\title{
FUSION CATEGORIES OF RANK 2
}

\author{
VIKTOR OSTRIK
}

\begin{abstract}
We classify semisimple rigid monoidal categories with two isomorphism classes of simple objects over the field of complex numbers. In the appendix written by P. Etingof it is proved that the number of semisimple Hopf algebras with a given finite number of irreducible representations is finite.
\end{abstract}

\section{Introduction}

Let $k$ be an algebraically closed field of characteristic 0 . A fusion category $\mathcal{C}$ over $k$ is a $k$-linear semisimple rigid monoidal category with finitely many simple objects and finite dimensional spaces of morphisms, such that the endomorphism algebra of the neutral object is $k$, see [3].

The problem of classification of all fusion categories seems to be very difficult. A basic invariant of a fusion category $\mathcal{C}$ is its Grothendieck ring $K(\mathcal{C})$ (physically, fusion rules algebra or fusion ring) which is a unital based ring in the sense of [10]. It is known that for a given based ring $K$ there are only finitely many fusion categories $\mathcal{C}$ with $K(\mathcal{C})=K$ (this statement is known as "Ocneanu rigidity," see [16] and [3]). There is a natural problem for a given "interesting" based ring $K$ to find all fusion categories $\mathcal{C}$ with $K(\mathcal{C})=K$ (such categories are called "categorifications" of $K$ ). This problem was considered first probably by G. Moore and N. Seiberg in [8], they considered the case when all objects of $\mathcal{C}$ are invertible and the case of Yang-Lee fusion rules (see below). In his thesis T. Kerler completely classified fusion categories with fusion rings isomorphic to the fusion ring of the category of integrable $\widehat{s l}_{2}$-modules at a positive integer level, see [4], and later D. Kazhdan and H. Wenzl generalized this to the case of $\widehat{s l}_{n}$, see [6]. In [13] D. Tambara and S. Yamagami considered another big class of examples, the so-called fusion rules of self-duality for finite abelian groups.

The rank of $K(\mathcal{C})$ over $\mathbb{Z}$ or equivalently the number of isomorphism classes of simple objects in $\mathcal{C}$ is called the rank of the category $\mathcal{C}$. There is only one fusion category of rank 1 - the category of vector spaces over $k$. In this note we will study all fusion categories of rank 2 . Let $\mathbf{1}, X$ be the simple objects of such category $\mathcal{C}$ (here $\mathbf{1}$ is the unit object). It is clear that possible fusion rules for $\mathcal{C}$ are completely determined by the number $n \in \mathbb{Z}_{\geq 0}$ from the equation $X \otimes X=\mathbf{1} \oplus n X$. Let $K_{n}$ denote the fusion ring corresponding to the number $n$.

Received May 14, 2002.

The author was partially supported by NSF grant DMS-0098830. 
The fusion ring $K_{1}$ is called the Yang-Lee fusion rules and is well known in the conformal field theory.

Main Theorem. There are just 4 fusion categories of rank 2. For two of them $K(\mathcal{C})=K_{0}$ and for other two of them $K(\mathcal{C})=K_{1}$.

The classification of fusion categories $\mathcal{C}$ with $K(\mathcal{C})=K_{0}$ or $K(\mathcal{C})=K_{1}$ is due to Moore and Seiberg [8]. The only new part of this Theorem is the fact that the fusion rings $K_{n}, n \geq 2$ admit no categorification.

Our result suggests that the answer to the following question is of interest:

Question. Is it true that there are only finitely many fusion categories of a given finite rank?

As a supporting evidence for positive answer to this question recall that the number of finite groups with a given number of irreducible representations is finite, see [7]. Moreover, P. Etingof proved that the number of finite dimensional semisimple Hopf algebras with a given number of irreducible representations is finite, see Appendix to this note (namely, Etingof gives a bound for the dimension of such Hopf algebra and Stefan's theorem [12] states that there are only finitely many semisimple Hopf algebras of a given dimension over an algebraically closed field of characteristic 0).

Remark. Our Main Theorem is not true without rigidity assumption on the category $\mathcal{C}$. See [14] for an example of a semisimple bialgebra with two representations and fusion rules $X \otimes X=2 X$.

\section{Proof of the Main Theorem}

It appears that direct methods developed in [13] are very difficult to apply in a case when some fusion coefficients are greater than 1 . In particular I could not study even the case of the fusion ring $K_{2}$ using these methods. So we are using another approach. Here is an outline of our proof. Let $\mathcal{C}$ be a fusion category with $K(\mathcal{C})=K_{n}$. First we study the Drinfeld double of $\mathcal{C}$ and show that $\mathcal{C}$ is braided. Then we show that the category $\mathcal{C}$ is automatically ribbon. It is easy to see then that the category $\mathcal{C}$ is modular (unless $n=0$ ). Now the standard identities from the theory of modular categories give us a contradiction.

2.1. The category $\mathcal{C}$ is braided. Let $Z(\mathcal{C})$ be the Drinfeld center of the category $\mathcal{C}$, see e.g. [9]. It is known that the category $Z(\mathcal{C})$ is semisimple, see [3]. Let $\mathcal{C}^{o p}$ denotes the opposite category to $\mathcal{C}$ and let $\mathcal{C} \otimes \mathcal{C}^{o p}$ be the external product of the categories $\mathcal{C}$ and $\mathcal{C}^{o p}$, see e.g. [9].

Lemma 2.1. The category $Z(\mathcal{C})$ has 4 simple objects $\mathbf{1}, X_{1}, X_{2}, Y$. Under the forgetful functor $F: Z(\mathcal{C}) \rightarrow \mathcal{C}$ one has $F(\mathbf{1})=\mathbf{1}, F\left(X_{1}\right)=F\left(X_{2}\right)=X, F(Y)=$ $X \otimes X$. 
Proof. Following [9] Theorem 4.14 (see also [11] Proposition 2.2) one can describe the category $Z(\mathcal{C})$ in the following way: the object $A=\mathbf{1} \otimes \mathbf{1} \oplus X \otimes X$ of the category $\mathcal{C} \otimes \mathcal{C}^{o p}$ has a natural structure of Frobenius algebra and the category $Z(\mathcal{C})$ is equivalent to the category of $A$-bimodules in $\mathcal{C} \otimes \mathcal{C}^{o p}$. Note that any (say left) $A$-module is free, that is of the form $A \otimes M$ where $M$ is some object of $\mathcal{C} \otimes \mathbf{1}$ (and $A$-module structure is the obvious one); this is a consequence of the general fact that the category of $A$-modules considered as the module category over $\mathcal{C} \otimes \mathcal{C}^{o p}$ is module equivalent to the category $\mathcal{C}$ with $X \otimes Y \in \mathcal{C} \otimes \mathcal{C}^{o p}$ acting via the functor $X \otimes ? \otimes Y$, see [11] (the equivalence sends an object $M \in \mathcal{C}$ to $A \otimes(M \otimes \mathbf{1}))$. So there are just two simple $A$-modules $-A$ itself and $A \otimes(X \otimes \mathbf{1})=\mathbf{1} \otimes X+X \otimes \mathbf{1}+n X \otimes X$.

We will denote by $\operatorname{Hom}(?, ?)$ the Hom-spaces in the category $\mathcal{C} \otimes \mathcal{C}^{o p}$ and by $\operatorname{Hom}_{A-A}(?, ?)$ the Hom-spaces in the category of $A$-bimodules. For any simple object $M \in \mathcal{C} \otimes \mathcal{C}^{o p}$ one considers the "free" bimodule $A \otimes M \otimes A$ (in other words, the object $A \otimes M \otimes A$ is just the image of $M$ under the functor $\mathcal{C} \rightarrow Z(\mathcal{C})$ left adjoint to the forgetful functor). Note that for any $A$-bimodule $B$ one has $\operatorname{Hom}_{A-A}(A \otimes M \otimes A, B)=\operatorname{Hom}(M, B)$. In particular any $A$-bimodule is a direct summand of some free bimodule. Now taking $M=\mathbf{1} \otimes \mathbf{1}$ we get that $\operatorname{Hom}_{A-A}(A \otimes A, A \otimes A)=\operatorname{Hom}(\mathbf{1} \otimes \mathbf{1}, A \otimes A)$ is two dimensional, so $A \otimes A$ is a direct sum of two nonisomorphic bimodules. One of them is $A$ itself, so $A \otimes A=A \oplus Y$ where the simple bimodule $Y$ as an object of $\mathcal{C} \otimes \mathcal{C}^{o p}$ has the following decomposition $Y=\mathbf{1} \otimes \mathbf{1} \oplus n \mathbf{1} \otimes X \oplus n X \otimes \mathbf{1} \oplus\left(n^{2}+1\right) X \otimes X$. This implies that for $M=\mathbf{1} \otimes X$ or $X \otimes \mathbf{1}$ one has $\operatorname{Hom}_{A-A}(A \otimes M \otimes A, Y)$ is $n$-dimensional and the calculation similar to the one above shows that $A \otimes M \otimes A=n Y \oplus X_{1} \oplus X_{2}$ where $X_{1}, X_{2}$ are simple nonisomorphic bimodules and $X_{1} \oplus X_{2}=21 \otimes X \oplus$ $2 X \otimes \mathbf{1} \oplus 2 n X \otimes X$. Since $X_{1}$ and $X_{2}$ are in particular $A$-modules one gets from the description of $A$-modules above that $X_{1}=X_{2}=\mathbf{1} \otimes X \oplus X \otimes \mathbf{1} \oplus X \otimes X$ as objects of $\mathcal{C} \otimes \mathcal{C}^{o p}$. Finally one calculates easily that for $M=X \otimes X$ one has $A \otimes M \otimes A=A \oplus n X_{1} \oplus n X_{2} \oplus\left(n^{2}+1\right) Y$ and so all $A$-bimodules are classified.

The forgetful functor $F$ has the following description on the objects of the category of $A$-bimodules: any $A$-bimodule $B$ is in particular left $A$-module, so is of the form $A \otimes(M \otimes \mathbf{1})$ where $M \in \mathcal{C}$; then $F(B)=M$. This finishes the proof of the Lemma.

The following Lemma calculates the fusion rules of $Z(\mathcal{C})$ and shows that the based ring $K(Z(\mathcal{C}))=K_{n} \otimes K_{n}$.

Lemma 2.2. We have

$$
X_{1} \otimes X_{1}=\mathbf{1} \oplus n X_{1} ; X_{2} \otimes X_{2}=\mathbf{1} \oplus n X_{2} ; X_{1} \otimes X_{2}=X_{2} \otimes X_{1}=Y .
$$

Proof. It is known that the category $Z(\mathcal{C})$ is rigid, see [9] Proposition 3.9. It is clear that $\mathbf{1}^{*}=\mathbf{1}$ and $Y^{*}=Y$. We claim that $X_{1}^{*}=X_{1}$ and $X_{2}^{*}=X_{2}$. Indeed otherwise $\operatorname{Hom}\left(\mathbf{1}, X_{1} \otimes X_{1}\right)=0$ and hence $X_{1} \otimes X_{1}=Y$ (since $\operatorname{Hom}\left(\mathbf{1}, F\left(X_{1} \otimes\right.\right.$ $\left.\left.X_{1}\right)\right) \neq 0$. Similarly $X_{2} \otimes X_{2}=Y$. We have also that $X_{1} \otimes X_{2}$ is a direct sum of 1 and $n$ summands each of which is isomorphic either to $X_{1}$ or to $X_{2}$. We can 
assume that $\operatorname{Hom}\left(X_{1} \otimes X_{2}, X_{1}\right) \neq 0$ (otherwise take $X_{1}$ instead of $X_{2}$ ). But in this case we have a contradiction:

$$
\begin{gathered}
0=\operatorname{Hom}\left(X_{1}, Y\right)=\operatorname{Hom}\left(X_{1}, X_{1} \otimes X_{1}\right)= \\
=\operatorname{Hom}\left(X_{1} \otimes X_{1}^{*}, X_{1}\right)=\operatorname{Hom}\left(X_{1} \otimes X_{2}, X_{1}\right) \neq 0 .
\end{gathered}
$$

Thus $X_{1}^{*}=X_{1}, X_{2}^{*}=X_{2}$ and hence $X_{1} \otimes X_{2}=X_{2} \otimes X_{1}=Y$. Note that

$$
\operatorname{Hom}\left(X_{1} \otimes X_{1}, X_{2}\right)=\operatorname{Hom}\left(X_{1}, X_{1}^{*} \otimes X_{2}\right)=\operatorname{Hom}\left(X_{1}, Y\right)=0
$$

so $X_{1} \otimes X_{1}=\mathbf{1} \oplus n X_{1}$. Similarly $X_{2} \otimes X_{2}=\mathbf{1} \oplus n X_{2}$. The Lemma is proved.

Thus the subcategory $\left\langle\mathbf{1}, X_{1}\right\rangle$ of $Z(\mathcal{C})$ consisting of direct sums of $\mathbf{1}$ and $X_{1}$ is a monoidal subcategory. The forgetful functor $F$ restricted to this subcategory is an equivalence of categories and thus we proved

Corollary 2.1. The category $\mathcal{C}$ admits a structure of braided category.

2.2. The category $\mathcal{C}$ is modular. Let us fix a structure of a braided category on $\mathcal{C}$. For $M, N \in \mathcal{C}$ let $\beta_{M, N}: M \otimes N \rightarrow N \otimes M$ denote the braiding morphism. The morphisms $\beta_{M, N}$ are completely determined by 4 morphisms $\beta_{M, N}$ where $M$ and $N$ are simple objects of $\mathcal{C}$ (since the braiding is functorial). It follows from the axioms that $\beta_{\mathbf{1 , 1}}=\mathrm{Id}, \beta_{\mathbf{1}, X}=\mathrm{Id}, \beta_{X, \mathbf{1}}=\mathrm{Id}$ so the only nontrivial morphism is $\beta_{X, X}$. The morphism $\beta_{X, X}$ induces a linear automorphisms of the one dimensional space $\operatorname{Hom}(\mathbf{1}, X \otimes X)$ and of the $n$-dimensional space $\operatorname{Hom}(X, X \otimes X)$; so the first is just some number $\mu \in k^{*}$ and the second is some linear operator $\Lambda$.

Lemma 2.3. One has $\Lambda^{2}=\mu I d$.

Proof. The vector space $\operatorname{Hom}(\mathbf{1}, X \otimes X \otimes X)$ carries the action of two linear operators $\Lambda_{1}:=\beta_{X, X} \otimes \operatorname{Id}$ and $\Lambda_{2}:=\operatorname{Id} \otimes \beta_{X, X}$ and it is enough to prove that $\Lambda_{1}^{2}=\mu \mathrm{Id}$. Note that by the hexagon axiom $\left(\operatorname{Id} \otimes \beta_{X, X}\right) \circ\left(\beta_{X, X} \otimes \mathrm{Id}\right)=\beta_{X, X} \otimes X$ and hence $\Lambda_{2} \Lambda_{1}=\mu \mathrm{Id}$. On the other hand the braid relation (= Yang-Baxter relation) says $\Lambda_{1} \Lambda_{2} \Lambda_{1}=\Lambda_{2} \Lambda_{1} \Lambda_{2}$ whence $\Lambda_{1}=\Lambda_{2}$ and the Lemma is proved.

Corollary 2.2. The category $\mathcal{C}$ admits a structure of ribbon (= pivotal and braided) category.

Proof. It is enough to define the twists by $\theta_{\mathbf{1}}=1$ and $\theta_{X}=\mu^{-1}$ (see [2] for notations).

Corollary 2.3. Assume that $n \neq 0$. Then the category $\mathcal{C}$ is modular.

Proof. It is easy to see that if $\mathcal{C}$ is not modular then $\mu=1$ and $\mathcal{C}$ is symmetric. But in a symmetric fusion category the dimensions of all objects are integers, see [1] Theorem 7.2. The Lemma is proved since the quadratic equation $x^{2}=1+n x$ has an integer root only for $n=0$. 
2.3. The category $\mathcal{C}$ does not exist. The main result of this note is the following:

Theorem 2.1. Assume that $n \geq 2$. There is no fusion category $\mathcal{C}$ such that $K(\mathcal{C})=K_{n}$.

Proof. We already proved that category $\mathcal{C}$ is modular if it exists. Let $d$ be a dimension of $X$ and let $\theta=\theta_{X}$. Thus $d$ is a root of equation $d^{2}=1+n d$ and $\theta$ is a root of unity by Vafa's Theorem, see [2] Theorem 3.1.19. Consider the Gaussian sums $p_{+}=1+\theta d^{2}$ and $p_{-}=1+\theta^{-1} d^{2}$. Since $\mathcal{C}$ is a modular category one has $p_{+} p_{-}=1+d^{2}$ (see [2] 3.1.15, 3.1.22) or, equivalently, $\theta+\theta^{-1}=-n d$. We can assume that $d>n$ (applying otherwise the Galois automorphism to the equation). Then $|n d|>n^{2}$ and $\left|\theta+\theta^{-1}\right| \leq 2$ and we have a contradiction for $n \geq 2$.

2.4. Categories $\mathcal{C}$ with $K(\mathcal{C})=K_{0}$. Let $G$ be a finite group and let $K(G)$ be the based ring with basis $X_{g}, g \in G$ and fusion rules $X_{g} \otimes X_{h}=X_{g h}$. It is shown in [8] Appendix E that the monoidal categories $\mathcal{C}$ with $K(\mathcal{C})=$ $K(G)$ are classified by $H^{3}\left(G, k^{*}\right)$. Any category of this kind is automatically rigid. In particular $K_{0}=K(\mathbb{Z} / 2 \mathbb{Z})$ and categorifications of $K_{0}$ are classified by $H^{3}\left(\mathbb{Z} / 2 \mathbb{Z}, k^{*}\right)=\mathbb{Z} / 2 \mathbb{Z}$. So there are two such categories. The first one is the category of representations of $\mathbb{Z} / 2 \mathbb{Z}$ and second differs from the first one by the sign of associativity morphism $(X \otimes X) \otimes X \rightarrow X \otimes(X \otimes X)$. This second category can be explicitly realized as the fusion category of integrable representations of $\widehat{s l}_{2}$ at level 1 .

2.5. Categories $\mathcal{C}$ with $K(\mathcal{C})=K_{1}$. Let $\mathcal{C}$ be a monoidal category with $K(\mathcal{C})=K_{1}$. Choose basis vectors in one dimensional vector spaces $\operatorname{Hom}(\mathbf{1}, X \otimes$ $X)$ and $\operatorname{Hom}(X, X \otimes X)$. The only nontrivial associativity morphism is $(X \otimes$ $X) \otimes X \rightarrow X \otimes(X \otimes X)$, this is equivalent to giving isomorphisms of vector spaces $\operatorname{Hom}(X, X \otimes X) \otimes \operatorname{Hom}(\mathbf{1}, X \otimes X) \rightarrow \operatorname{Hom}(X, X \otimes X) \otimes \operatorname{Hom}(\mathbf{1}, X \otimes X)$ (so this is just a number $\lambda \in k^{*}$ ) and $\operatorname{Hom}(\mathbf{1}, X \otimes X) \otimes \operatorname{Hom}(X, X \otimes \mathbf{1}) \oplus$ $\operatorname{Hom}(X, X \otimes X) \otimes \operatorname{Hom}(X, X \otimes X) \rightarrow \operatorname{Hom}(\mathbf{1}, X \otimes X) \otimes \operatorname{Hom}(X, \mathbf{1} \otimes X) \oplus$ $\operatorname{Hom}(X, X \otimes X) \otimes \operatorname{Hom}(X, X \otimes X)$ (so this can be represented by invertible $2 \times 2$ matrix). After relatively easy calculation (along the lines of [13]) one finds that $\lambda=1$ and the second isomorphism can be represented by the matrix $\left(\begin{array}{cc}a & 1 \\ a & -a\end{array}\right)$ where $a$ is a root of equation $a^{2}+a=1$. So we have two solutions; both categories are rigid; they differ by the action of Galois group. In one of these categories $\operatorname{dim}(X)=\frac{1+\sqrt{5}}{2}$ and in the second $\operatorname{dim}(X)=\frac{1-\sqrt{5}}{2}$. The first category can be explicitly realized as a subcategory of "integer spin" representations of the fusion category of integrable $\widehat{s l}_{2}$-modules at level 3 and the second category is the minimal model $\mathcal{M}(2,5)$ for the Virasoro algebra (with central charge $c=-\frac{22}{5}$ ). Both categories can be also realized using the quantum group $U_{q}\left(s l_{2}\right)$ for $q=\sqrt[10]{1}$ (there are 4 primitive tenth roots of 1 , but $q$ and $q^{-1}$ give rise to the same category), see [2]. 


\section{Acknowledgement}

I am much obliged to Tania Chmutova, Pavel Etingof and Dmitri Nikshych for useful discussions; many thanks are due to Pavel Etingof for writing the Appendix to this note. I am grateful to Richard Stanley for providing reference [7].

\section{Appendix}

\section{Pavel Etingof}

In this appendix we give an upper bound for the dimension of a semisimple Hopf algebra over $k$ with $n$ irreducible representations. For group algebras, this estimate was obtained 100 years ago by E. Landau, [7]. (We are grateful to R. Stanley for giving us this reference).

For a positive integer $n$, let $P(n)$ be the set of positive integer solutions of the equation $\frac{1}{x_{1}}+\ldots+\frac{1}{x_{n}}=1$, and let $d(n)=\max _{\left(x_{1}, \ldots, x_{n}\right) \in P(n)} \max _{i} x_{i}$.

Theorem 3.1. Let $H$ be a semisimple Hopf algebra over $k$, which has $n$ irreducible representations. Then $\operatorname{dim} H \leq d(n)$.

Remark 1. The set $P(n)$ is finite, since for any positive rational number $r$ the number of positive integer solutions of the equation $\frac{1}{x_{1}}+\ldots+\frac{1}{x_{n}}=r$ is finite ([7]). Indeed, if $x_{1}$ is the smallest coordinate, then $x_{1} \leq n / r$, so there are finitely many possibilities for $x_{1}$, and if $x_{1}$ is fixed then $\left(x_{2}, \ldots, x_{n}\right)$ vary over the set of positive integer solutions of the equation $\frac{1}{x_{2}}+\ldots+\frac{1}{x_{n}}=r-\frac{1}{x_{1}}$, so the statement follows by induction. (In fact, this inductive procedure allows one to obtain an explicit estimate for $d(n)$, see [7]).

Remark 2. One has $d(n+1) \geq 2 d(n)$ since if $\sum_{i=1}^{n} \frac{1}{x_{i}}=1$ then $\sum_{i=1}^{n} \frac{1}{2 x_{i}}+\frac{1}{2}=$ 1 .

Proof. Our proof is a generalization of the classical proof of Landau in the group case, which employs the fact that for a group with $n$ irreducible representations, one has $\sum_{i=1}^{n} \frac{1}{\left|C_{i}\right|}=1$, where $C_{i}$ are centralizers of conjugacy classes (the class equation). Namely, we use the Hopf algebraic class equation, due to G.Kac [5] and Y.Zhu [15].

Let $C(H) \subset H^{*}$ be the character ring of $H$ (it is spanned by characters of irreducible representation). If $H$ is semisimple then $C(H)$ is a semisimple algebra (see [15], Lemma 2) of dimension $n$, so $C(H)$ can be identified with $\oplus \mathrm{Mat}_{r_{i}}$, where $\sum r_{i}^{2}=n$. Let us choose such an identification, and let $E_{j j}^{(i)} \in C(H)$ be the corresponding matrix units.

Theorem (Kac-Zhu). [15] The ratio $m_{i}=\operatorname{dim}(\mathrm{H}) /\left.\operatorname{Tr}\right|_{H^{*}}\left(E_{j j}^{(i)} \cdot\right)$ (where for $a \in$ $H^{*} a \cdot$ denotes the operator of multiplication by a) is a positive integer.

Now observe that $\sum_{i} \sum_{j=1}^{r_{i}} E_{j j}^{(i)}=1$, so $\sum_{i} \frac{r_{i}}{m}=\sum_{i} \sum_{j=1}^{r_{i}} \frac{1}{m_{i}}=1$. Writing $r_{i} / m_{i}$ as a sum of $r_{i}$ copies of $1 / m_{i}$, we get a solution of the equation 
$\sum_{j=1}^{r} \frac{1}{x_{i}}=1$, where $r=\sum_{i} r_{i} \leq n$. Thus, $m_{i} \leq d(r) \leq d(n)$. On the other hand, consider the 1-dimensional matrix block of $C(H)$ spanned by the integral of $H^{*}$ (i.e. by the character of the regular representation of $H$ ). For this block the number $m_{i}$ is obviously equal to $\operatorname{dim}(H)$, since the integral is a projector to a 1-dimensional subspace. So $\operatorname{dim} H \leq d(n)$, as desired.

\section{References}

[1] N. Andruskiewitsch, P. Etingof, S. Gelaki, Triangular Hopf algebras with the Chevalley property, Michigan Math. J. 49 (2001), 277-298.

[2] B. Bakalov, A. Kirillov Jr., Lectures on tensor categories and modular functor, University Lecture Series, 21. American Mathematical Society, Providence, RI, 2001.

[3] P. Etingof, D. Nikshych, V. Ostrik, On fusion categories, preprint: math.QA/0203060

[4] J. Frölich, T. Kerler, Quantum groups, quantum categories and quantum field theory, Lecture Notes in Mathematics, 1542. Springer-Verlag, Berlin, 1993.

[5] G. I. Kac, Certain arithmetic properties of ring groups, (Russian) Funkcional. Anal. i Priložen. 6 (1972), 88-90.

[6] D. Kazhdan, H. Wenzl, Reconstructing monoidal categories, I. M. Gelfand Seminar, 111136, Adv. Soviet Math., 16, Part 2, Amer. Math. Soc., Providence, RI, 1993.

[7] E. Landau, Über die Klassenzahl der binären quadratischen Formen von negativer Discriminante, Math. Ann. 56 (1903), 671-676.

[8] G. Moore, N. Seiberg, Classical and quantum conformal field theory, Comm. Math. Phys. 123 (1989), 177-254.

[9] M. Müger, From subfactors to categories and topology. II, preprint: math. CT/0111205

[10] V. Ostrik, Module categories, weak Hopf algebras and modular invariants, preprint: math.QA/0111139

[11] _ Boundary conditions for holomorphic orbifolds, preprint: math.QA/0202130

[12] D. Stefan, The set of types of n-dimensional semisimple and cosemisimple Hopf algebras is finite, J. Algebra 193 (1997), 571-580.

[13] D. Tambara, S. Yamagami, Tensor categories with fusion rules of self-duality for finite abelian groups, J. Algebra 209 (1998), 692-707.

[14] E. Vaysleb, Cosemisimple bialgebras and discrete quantum semigroups, J. Algebra 197 (1997), 506-520.

[15] Y. Zhu, Hopf algebras of prime dimension, Internat. Math. Res. Notices 1994, 53-59.

[16] A. Wassermann, Quantum subgroups and vertex algebras, talk given at MSRI, available at http://www.msri.org/publications/ln/msri/2000/subfactors/wassermann/1/

Department of Mathematics, Mit, 77 Massachusetts Ave., Cambridge, MA 02139, U.S.A.

E-mail address: ostrik@math.mit.edu 